\title{
Low Voltage Power Line Noise Compression Algorithm Simulation Based on the Compression Sensing
}

\author{
Xie Yan ${ }^{1, a}$, Yang Guang ${ }^{2, ~ b}$, Dapeng Lin ${ }^{3, c}$, Tailin $\mathrm{Wu}^{4, \mathrm{~d}}$ and Shouyang Long ${ }^{5, \mathrm{e}}$ \\ ${ }^{1,2}$ State grid Tianjin electric power company, Tianjin, China \\ ${ }^{3,4,5}$ China GRIDCOM Co., Ltd ,Shenzhen ,Guangdong, China \\ aXie_yan_xy@sina.com.cn, ${ }^{\mathrm{b}}$ Capshine@126.com, ${ }^{\mathrm{e}}$ longshouyang@sgitg.sgcc.com.cn
}

Keywords: Power Line Communication, Noise Compression Algorithm, Compression Sensing Algorithm, Channel Attenuation, Noise Acquisition, Background Noise, Random Noise.

\begin{abstract}
Noise is one of the most main factors that affect the reliability of the power line communication; its time-varying characteristic is very strong, so noise acquisition and efficient storage is a must. But now, the noise sampling rate is too high, only a few hours, the amount of data storage may reach $\mathrm{T}$ level, in this way cost is too high. This article presents a noise compression storage method, which can effectively compress noise collected from the different time and location, in this way we can greatly reduce the data storage; it will provide a lot of help to the noise analysis and testing in the laboratory later.
\end{abstract}

\section{Introduction}

The power line is designed to transmit electricity, not specially used for data transmission communication, its topology structure and physical properties are different from conventional transmission medium of communication ${ }^{[1]}$. Due to the existence of bad power line communication environment, such as channel attenuation, the noise interference and electromagnetic compatibility, the power line communication is seriously affected by time, frequency and load.

The noise is one of the most main factors that affect the reliability of the power line communication, its time-varying characteristic is very strong, and so noise acquisition and efficient storage is a must. But now the existing noise recording equipment only work in power line no-load ${ }^{[2]}$, the collected noise need to be stored, but because the sampling rate is too high, only a few hours, the amount of data storage will reach $\mathrm{T}$ level, in this way cost is too high.

In order to make kinds of PLC equipment apply to the power line communication network, we urgently need a compression storage method, which can effectively compress noise from the different time and location, in this way we can greatly reduce the data storage, it will provide a lot of help to the noise analysis and testing in the laboratory later.

\section{Low voltage power line channel and noise characteristics}

The low voltage power line is mainly used for the $50 / 60 \mathrm{~Hz}$ power distribution in a short distance, so compared with dedicated communication line, the power line channel environment is extremely bad, mainly include ${ }^{[4]}$ :

(1)Meet disturbance signal in wide range. Such as all kinds of electrical equipment, especially the old and having quality defects equipment, will bring disastrous interference to the power line communication.

(2)The impedance of the power network has large variation along with load changes, and it also has strong time variability.

(3)The strong attenuation characteristic makes the various nodes show the different performance on the power line

The main factors influencing the reliability of the power line communication are: the high noise level, the large variation impedance, signal attenuation intense. So the noise, impedance and 
attenuation need to consider for channel modeling. The noise characteristic is mainly analyzed and discussed in this paper.

At present, five types of noise are recognized in the low voltage power line communication ${ }^{\text {[2] [5], }}$, they are, respectively, the colored background noise, narrow-band noise, periodic impulse noise synchronized to the power frequency, periodic impulse noise asynchronous to the power frequency and asynchronous aperiodic impulse noise. These noise distributions are closely related to the time, place and load, which makes power line noise have strong time-varying characteristics, at the same time, due to the wide spectrum of power line noise, according to the laws of Nyquist sampling, A/D sampling rate must be more than twice the highest noise. Collection power line noise, therefore, need a high sampling rate, for ordinary power line noise acquisition device, it is difficult to complete effective storage for a long time these large amounts of data.

Because the sampling rate is too high, only a few hours, the amount of data storage will reach $\mathrm{T}$ level, in this way cost is too high. Therefore, a new power line noise acquisition compression algorithm becomes very meaningful.

\section{Compression sensing model}

The traditional compression algorithm is shown in Fig.1:

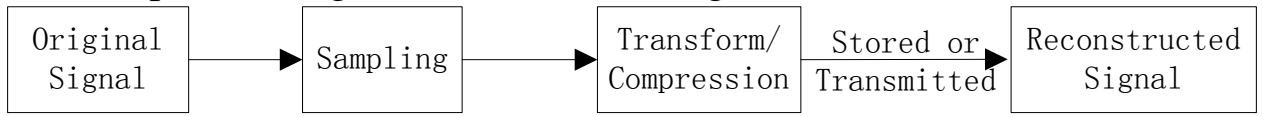

Fig.1 The traditional compression algorithm process

The compressed sensing algorithm process is shown in Fig.2:

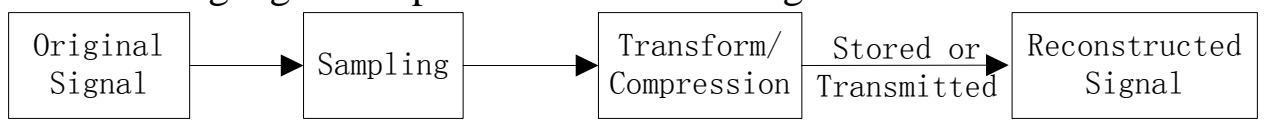

Fig.2 The compressed sensing algorithm process

Specific theory principle is as follows:

Suppose a one-dimensional finite discrete signal $x \in R^{N \times 1}$, as a column vector $R^{N}$, because the space of all $R^{N}$ signals can be made by a group $\psi=\left[\phi_{1}, \phi_{2}, \ldots \ldots, \phi_{\mathrm{N}}\right]$ of a linear combination, assuming these base vector is standard orthogonal ${ }^{[7]}, x$ can be represented as formula (1), namely:

$$
x=\sum_{i=1}^{N} \phi_{i} \alpha_{i}=\psi \alpha .
$$

In the formula: $\alpha_{\mathrm{i}}=\left\langle x, \phi_{i}\right\rangle$ is the projection coefficient, $\alpha=\psi^{T} x$ is a projection coefficient vector, $\psi=\left[\phi_{1}, \phi_{2}, \ldots \ldots, \phi_{\mathrm{N}}\right]$ is orthogonal basis matrix, $\alpha$ and $x$ are $N \times 1$ column vectors, $\psi$ is $N \times N$ matrix.

From the above, $x$ is the representation of signal in time domain, $\alpha$ is the representation of signal in the $\psi$ domain, they are equivalent. The theory of compressed sensing indicated: As long as the number of the signal on a base of non-zero coefficient (or greater than zero) $\mathrm{K}<<\mathrm{N}$, according to the signal $x$ on the orthogonal basis $\psi$ is sparse or compressible, the sparse degree is K. Formula (1) is sparse representation of signal, $\psi$ is sparse basis.

If $x$ meets the condition of sparse or compressible, we can observe signals by using the observation matrix $\Phi$ that is not related to transform radix, and the observation vector $y$ is obtained.

$y=\Phi x$

Here, $\Phi$ is $M \times N(M \triangleleft \triangleleft N), y$ is $M \times 1$, It seems from Formula(2) that the observation sequence dimensions $\mathrm{M}$ after projection is far less than the original signal dimension $\mathrm{N}$, that is to say, the signal has been greatly compressed, observation sequence data volume is far less than the data sources from the Nyquist sampling. 
The theory of compressed sensing indicated ${ }^{[7]}$ : when the observation matrix $\Phi$ satisfies condition constraint equidistant and irrelevant features, $x$ is $K$ sparse and $K \triangleleft M \triangleleft \triangleleft N$, we can get Sparse coefficient $\alpha$ by solving optimization problem of 0 norm significance, general use formula (3):

$\left(L_{0}\right) \alpha=\min \|\alpha\|_{0} \quad$ s.t $\quad y=\Phi x=\Phi \psi \alpha$

Original signal is obtained by solving the sparse coefficient $\alpha$ and plugging $\alpha$ into formula (3).

\section{The application of compressed sensing algorithm in the noise acquisition}

The application of compressed sensing in the noise acquisition includes the following steps:

(1) When the power line is no-load, the noise is isolated and attenuated by the coupler, again it will be high sampled by the noise acquisition device, and it will be used to compress storage;

(2) Segmenting the collected noise, for the random noise sparse said:

Through the noise analysis, the five kinds of noise are divided into the following two types:

The first type is the background noise, including the colored background noise, narrowband noise and asynchronous periodic impulse noise, this three kinds of noise change slowly over time, the average power is small, but the spectrum is wide, and continue to exist, may have some or all of the cover signal spectrum.

The second type is a random noise, it includes the synchronous with power frequency of periodic and stochastic pulse noise, two kinds of the non-stationary noise is strong, when these noise power spectral density will rise suddenly, data transmission will cause a great error.

Among them, the background noise has the characteristics of the smaller average power, wide spectrum, is similar to white noise, but the random noise with strong time-varying is suddenly unpredictable, so two types of noise will be separated in the frequency domain by determining threshold, so that the random noise is sparse;

The overall schematic diagram is as Fig.3:

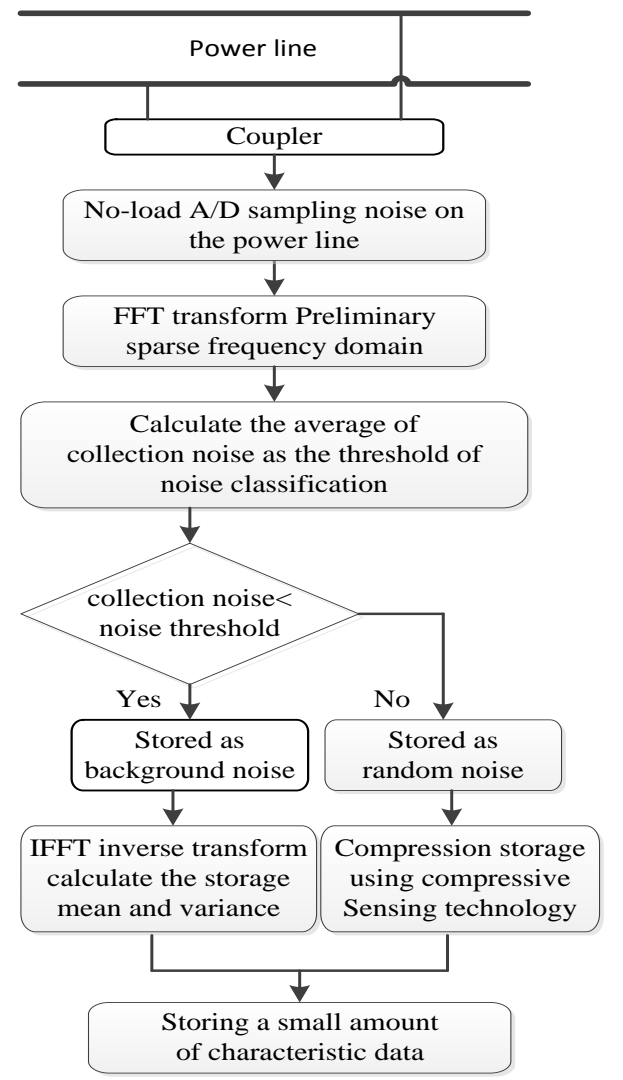

Fig.3 The diagram of compressed sensing

The concrete process of the sparse steps as follows:

a)The collected noise can be random noise of sparse said on the frequency domain through analysis, so first FFT transform for the collected noise, and get the noise frequency domain said; 
b)Because of the high sampling rate, the collected noise will get a lot of time domain data points, in order to reduce the amount of data processing, preliminary noise of thinning processing, namely the regular extraction of data points collected, and reduce the amount of data;

c)Calculating the median number of sampling points for noise, and getting two kinds of noise classification decision threshold;

d)Compared with the initially sparsely noise and the decision threshold: if the acquisition of the noise amplitude is less than the threshold, it is classified as background noise; if not, as random noise, and this random noise is sparse.

(3)On the one hand, for the background noise, it is necessary for feature extraction from time domain, the main parameters including the mean and variance, and storing these characteristic parameters can achieve the purpose of reducing noise recording storage;

On the other hand, for the random noise, it is compressed sensing from frequency domain, the appropriate observation matrix is constructed, the main information of this noise is extracted, that is to say, there is relatively large values of data points for storage, removing most of point data for the zero amplitude, storing the useful information. It will greatly save the storage space by using these two kinds' methods for noise data characteristics storage.

To take advantage of the compressed sensing technology to compress storage for random noise, it is necessary to construct the appropriate observation matrix ${ }^{[3]}$, which must meet $\mathrm{K}$ step restrict isometric property limited isometric properties, namely:

$$
\left(1-\delta_{k}\right)\|x\|_{2}^{2} \leq\|\phi x\|_{2}^{2} \leq\left(1+\delta_{k}\right)\|x\|_{2}^{2} \quad \delta_{k} \in(0,1)
$$

The common observation matrix has three types: Gaussian/sub-Gaussian/Bernoulli stochastic observation matrix, Fourier observation matrix and other observation matrix.

Gaussian random observation matrix is used most commonly; its concrete construction method is that making each element $\phi_{i, j}$ of the matrix $\phi \in R^{M \times N}(N \ll N)$ independently obeys the $N(0,1 / \sqrt{M})$ distribution. Gaussian random matrix is not related with most of the fixed orthogonal basis, when $M \geq c K \log (N / K)$ ( $c$ is a very small constant), it may get $\theta=\phi \varphi^{T}$, this can meet the high probability restrict isometric property.

The isolated random noise $x$ compressed sensing from the frequency domain:

$$
\left[\begin{array}{c}
y_{1} \\
y_{2} \\
\vdots \\
y_{M-1} \\
y_{M}
\end{array}\right]_{M \times 1}=\left[\begin{array}{ccc}
\theta_{1} & & \theta_{N} \\
\theta_{2} & \ddots & \theta_{N+1} \\
\vdots & & \vdots \\
\theta_{M-1} & \ddots & \theta_{N+M-1} \\
\theta_{M} & & \theta_{N+M}
\end{array}\right]_{M \times N}\left[\begin{array}{c}
x_{1} \\
x_{2} \\
\vdots \\
x_{N-1} \\
x_{N}
\end{array}\right]_{N \times 1}
$$

In this way, we obtain the noise main information, which have a larger amplitude data points storage, remove most of the data points to zero amplitude, that is, only store the useful information is Analog-to-Information Conversion of process, can achieve the purpose of reducing the amount of noise storage.

\section{Algorithm Simulation}

\section{(1)Simulation conditions}

Establishing power line noise model, which is synthesized by five types of noise.

(2)Simulation content

Five kinds of noise and complex noise are generated according to the power line channel noise classification, they are used for the subsequent studies and verification about compressed sensing technology and noise storage.

Among them, the colored background noise can be simply synthesized by a white noise source filtering; narrowband noise can be described by superposed of $\mathrm{N}$ independent sine functions; the 
periodic impulse noise, synchronized to the power frequency, is mainly generated by a silicon controlled rectifier conditioning device; the sources of impulse noise asynchronous to the power frequency has two aspects, one is the line frequency scanning and its harmonics of television monitor and computer displayer, the other is a periodic pulse noise generated by the computer switching power supply.

After generating five typical power line noises and they resultant noise, data amount of the high rate $\mathrm{A} / \mathrm{D}$ sampled noise is very large, you can use the compressed sensing algorithm proposed to be effective compression and storage, and solve the inconvenience of storing data due to the large amount of data.

(3) Simulation Results

Fig 4 shows the original background noise frequency response in a typical power line communication system, because the colored background noise, narrow-band noise and periodic pulse-frequency induction noise change slowly over time, they are often attributed to the background noise. Through the noise capture device acquisition and analysis, separating out background noise and storing the effective characteristic parameters mean and variance.

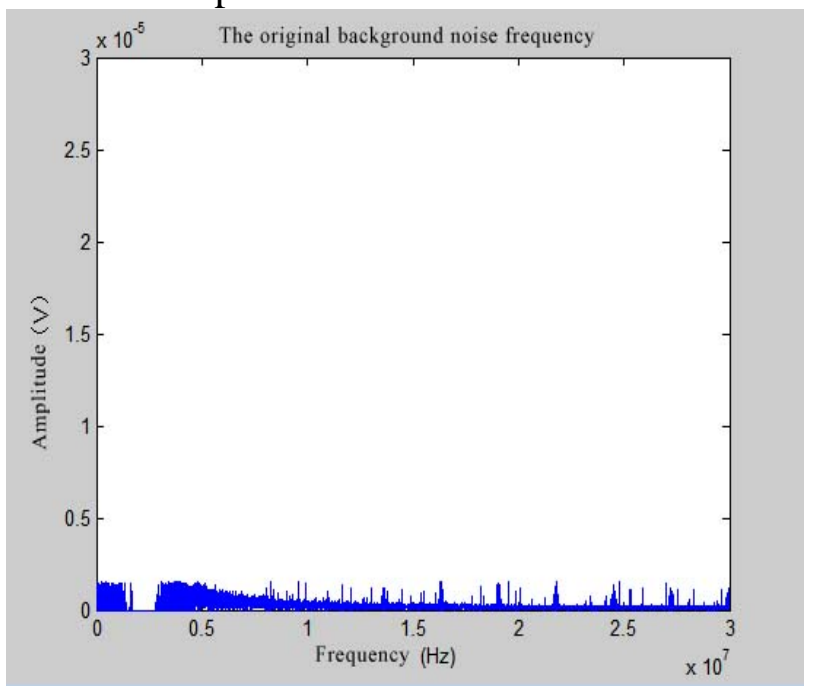

Fig.4 The original background noise frequency response

Fig.5 shows that in a typical power line communication system, since the frequency synchronization when periodic pulse noise and random impulse noise strong variability, when there is the noise power spectral density will suddenly rise, data transmission could be very a large error, often attributed to random noise, by contrast with the decision threshold of the random noise in the frequency domain data separated.

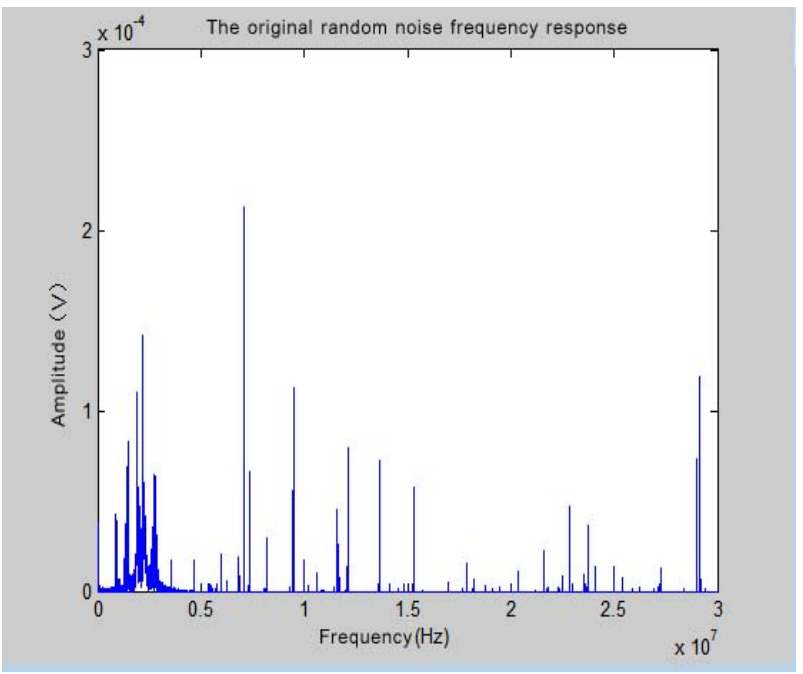

Fig.5 The original random noise frequency response

Shown in Fig.6, the article proposed compressed sensing method for the sparsity of random noise of the compression process, only a small number of features to store information. 


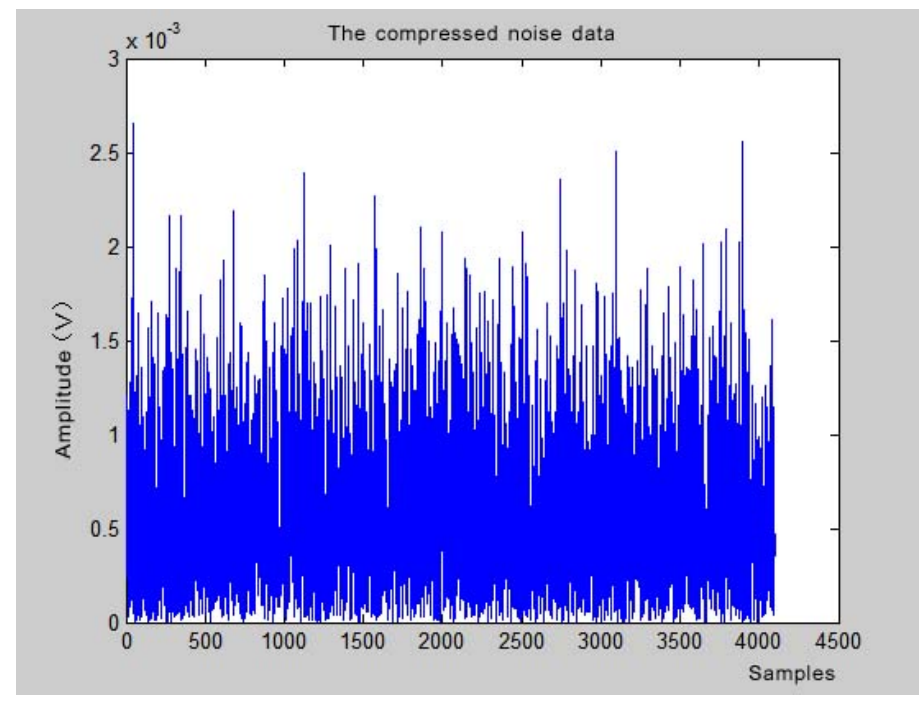

Fig.6 The compressed noise data

Simulation results show that the present invention with the current power line noise recording and playback device compared to impulse noise detection method, since the present invention considers the problem of data collection and processing time, to collect data for a reasonable compression and storage, reducing storage data share resources, and its effectiveness to be far better than the power line noise recording and playback device, impulse noise detection method.

\section{Conclusions}

In this paper, a more effective compression method for power line noise is proposed, which makes full use of the current compressed sensing technology, and can acquire noise when the power line is no-load. And the existing power line noise acquisition and recording equipment can only collect and store a short of the noise with large memory. So this method in the paper provides a new method and idea for solving the effective compression storage of massive power line noise data

\section{References}

[1] Gotz M, Rapp M, Dostert K. Power line channel characteristics and their effect on Communication system design [J].Communications Magazine, IEEE, 2004, 42(4):78-86.

[2] Meng H, Guan Y L, Chen S. Modeling and analysis of noise effects on broadband power-line communications [J]. Power Delivery, IEEE Transactions on, 2005, 20(2): 630-637.

[3] Sancha S, Canete F J, Diez L, et al. A channel simulator for indoor power-line communications[C]// Power Line Communications and Its Applications, 2007. ISPLC'07. IEEE International Symposium on. IEEE, 2007: 104-109.

[4] Zhang S Q, Yu D H. The measurement and analysis of noise characteristic in low voltage power line communication channel [J].Telecommunications for Electric Power System,2003, 24(1): $35-38$

[5] Lin H, Lin D.Modeling of PLC Noise and Design of Noise Generator[J]. Telecommunications for Electric Power System.2012, 25(5): 34-37.

[6] Zhang $\mathrm{H}$ X,Zhang $\mathrm{X}$ M,Tai X.Noise analysis and modeling research in power line channel[J].Journal of Chongqing University of Posts and Telecommunications (Natural Science Edition).ISTIC, 2013, 25(5).

[7] Peng Yulou, He Yigang, Lin Bin. Noise signal recovery algorithm based on singular value decomposition in compressed sensing [J].Chinese Journal of Scientific Instrument. 2012,12(33): 2655-2660. 